\title{
The Additional Value of Free Prostate Specific Antigen to the Battery of Age-Dependent Prostate-Specific Antigen, Prostate-Specific Antigen Density and Velocity
}

\author{
Mira Barak ${ }^{1}$, Michael Cohen ${ }^{3}$, Yoel Mecz ${ }^{3}$,Avi Stein ${ }^{3}$, Roman Rashkovitzki ${ }^{3}$, Benjamin Laver ${ }^{2}$ and Aharon Lurie ${ }^{3}$ \\ ${ }^{1}$ Kupat Holim Clalit, Biochemistry Department, Haifa and Western Galilee Central Laboratory, Nesher, Israel \\ 2 Urology Department, Lin Medical Center, Haifa, Israel \\ ${ }^{3}$ Urology Department, Carmel Medical Center, Haifa, Israel
}

Summary: This study describes the value of using the fraction of free prostate-specific antigen as a further marker in the early detection of prostate cancer. This newly introduced marker is compared to the usual battery of agedependent total prostate-specific antigen, prostate-specific antigen density $(\mu \mathrm{g} / 1 \times \mathrm{g}$ tissue) and prostate-specific antigen velocity $(\mu \mathrm{g} / 1 \times$ year $)$.

Determination of total prostate-specific antigen and free prostate-specific antigen was performed on fresh serum samples obtained from 3470 symptomatic patients aged 45-80 attending the Urology Clinics, or their General Practitioners. Among them, 310 patients had total prostate-specific antigen above the age-dependent cut-off, and/or free/total prostate-specific antigen under $11 \%$, with different prostate-specific antigen densities and velocities. Only 147 patients complied to undergo biopsy: in 72 of those patients, benign prostatic disease was histologically confirmed, while in 75 patients primary prostate cancer was histologically confirmed. Total and free prostate-specific antigen levels were determined using the third generation DPCs prostate-specific antigen assay performed on the Immulite automated immunoassay instrument. Total prostate-specific antigen age reference values were adopted from Oesterling et al. (J Am Med Ass 1993; 270:860-4); the prostate-specific antigen density was considered suspicious of prostate cancer if it was greater than $0.15 \mu \mathrm{g} / 1$ prostate-specific antigen per gram tissue (Seaman et al. Urol Clin N Am 1993; 20:653); prostate-specific antigen velocity greater than $0.75 \mu \mathrm{g} / 1 \times$ year $($ Carter et al., $\mathrm{J}$ Am Med Ass 1992; 267:215) was considered suspicious for prostate cancer.

Of the 147 patients, 75 had prostate cancer and 72 had benign prostatic hypertrophy. The difference between prostate cancer and benign prostatic hypertrophy was significantly reflected only by free/total prostate-specific antigen and prostate-specific antigen velocity. These parameters also provided the best sensitivity and specificity. Only these parameters proved to be significant when using a backwards logistic regression model (prostate-specific antigen velocity, $\mathrm{p}=0.007$ odds ratio 2.782 ; free/total prostate-specific antigen $\%, p=0.016$ odds ratio 2.678 ). Combinations of various parameters became significant when including free/total prostate-specific antigen, increasing prostate cancer detection to $88 \%$.

We conclude that free/total prostate-specific antigen is the most significant among prostate-specific antigen quantities (total age-dependent prostate-specific antigen, prostate-specific antigen density and prostate-specific antigen velocity). Adding this parameter to other prostate-specific antigen parameters improves the discrimination between prostate cancer and benign prostatic hypertrophy for the population at risk.

\section{Introduction}

The benefit of early detection of prostate cancer is controversial (2-6). However, until appropriate studies are conducted to determine the population at risk and on the individual level the patient who will benefit from early detection, clinicians are obligated to provide the patient with all possible modes of diagnosis and treatment, together with the basic knowledge on the natural history of the disease. The use of prostate-specific antigen as a tool in the diagnosis of prostate cancer has gained wide acceptance because about $70 \%$ of patients with cancers identified by digital rectal examination, transrectal ultra- sound and needle biopsy have prostate-specific antigen concentrations above the cut-off values used $(7-11)$. Prostate-specific antigen alone will miss $38-48 \%$ of organ-confined but clinically significant prostate cancer $(12-13)$. On the other hand, approximately only one in four men with prostate-specific antigen above $4 \mu \mathrm{g} / \mathrm{l}$ will have a prostate cancer $(8,9,11,14)$. The detection of prostatic cancer in its curable stages requires the use of relatively low prostate-specific antigen cut-off, since low cut-offs render high false positive rates, leading to unnecessary biopsies. Prostate-specific antigen density 
$(15-17)$, prostate-specific antigen velocity $(18,19)$ and prostate-specific antigen age-specific reference ranges $(1,20-22)$ were introduced in order to improve the clinical use of prostate-specific antigen for early detection of prostate cancer. Another potential way for refinement of the prostate-specific antigen assay was the introduction of the free prostate-specific antigen test. Several investigators have demonstrated that prostate-specific antigen is complexed (in vitro) to the protease inhibitors $\alpha_{1}$-antichymotrypsin and $\alpha_{2}$-macroglobulin $(23-25)$ : the commercial assays for prostate-specific antigen measure complexed as well as free prostate-specific antigen, and most of them are claimed to measure equimolar quantities of both (26). The proportion of free prostatespecific antigen is lower in prostate cancer than in benign prostatic hypertrophy and the free/total prostatespecific antigen ratio can distinguish better between prostate cancer and benign prostatic hypertrophy $(27-31)$. In this study we measured the free/total prostate-specific antigen and correlated it to the battery consisting of age-adjusted total prostate-specific antigen, prostate-specific antigen density and prostate-specific antigen velocity usually used in our laboratory for detection of prostate cancer.

\section{Materials and Methods}

\section{Subjects and procedures}

From February 1995 through December 1995, we measured prospectively total prostate-specific antigen and free prostate-specific antigen concentrations in 3470 men aged 45 to 80 years $(67.3 \pm 7.5$ years, mean \pm S. D.). All these patients complained of benign prostatic hypertrophy-related urinary symptoms (nocturia, hesitancy, incomplete voiding sensation, etc.). Patients with a previous history of prostate cancer, prostatitis or urinary infections were excluded. Blood was drawn prior to the digital rectal examination and transferred promptly to the laboratory. After 30 minutes at room temperature, the samples were centrifuged for 10 minutes at $1500 \mathrm{~g}$, the serum was removed and assayed for both total and free prostate-specific antigen. In a pilot study performed on 100 men using a ROC (receiver operating characteristic) curve analysis, we found a cut-off value of $11 \%$ for free/total prostate-specific antigen (see Results) to give best discrimination between benign prostatic hypertrophy and prostate cancer. Of the 310 patients having prostate-specific antigen levels higher than the age-related cut-off or those having free/total prostate-specific antigen lower than $11 \%$ were asked to repeat both tests within one month. Of these, 147 patients having repeated positive tests were referred to our institute for further evaluation. They underwent digital rectal examination and prostatic trans-rectal ultrasound (see flow chart, fig. 1). The age reference prostate-specific antigen concentrations used were:

$\leq 49$ years - prostate-specific antigen $\leq 2.5 \mu \mathrm{g} / \mathrm{l}$,

$\leq 59$ years - prostate-specific antigen $\leq 3.5 \mu \mathrm{g} / \mathrm{l}$,

$\leq 69$ years - prostate-specific antigen $\leq 4.5 \mu \mathrm{g} / \mathrm{l}$,

$\leq 79$ years - prostate-specific antigen $\leq 6.5 \mu \mathrm{g} / \mathrm{l}$.

Prostate-specific antigen density was calculated by dividing the total prostate-specific antigen level by the prostate volume, which was calculated via the prolate spheroid formula using the transrectal ultrasound scan (32). A density higher than $0.15 \mu \mathrm{g} / 1 \times \mathrm{g}$ (17) was considered suspicious for prostate cancer. Prostate-specific antigen velocity was calculated by dividing the difference between two consecutive prostate-specific antigen determinations performed during 3 to 12 months divided by the period between

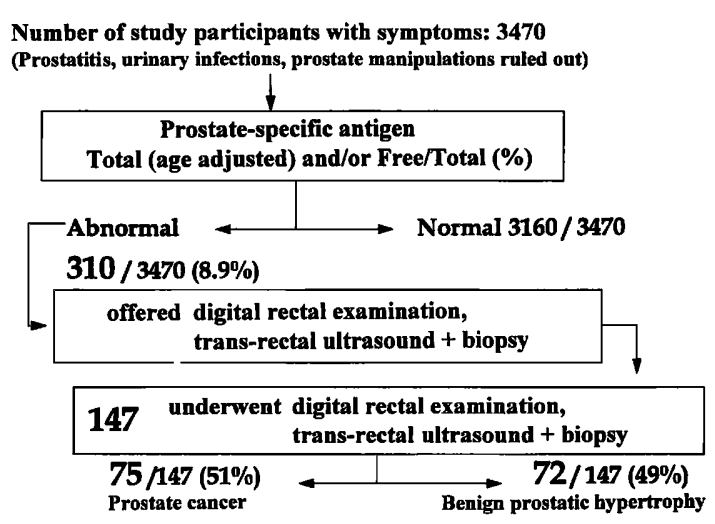

Fig. 1 Patients flow chart. The initial study comprised 3470 symptomatic patients (any previous non-related prostatic or urinary disease or manipulation was ruled out). Threehundred-ten of them had abnormal age-related total prostate-specific antigen and/or abnormal free/total prostate-specific antigen and were offered further evaluation. Hundred-fourty seven patients underwent digital rectal examination, trans-rectal ultrasound and prostate biopsies (core). Seventy-five patients had prostate cancer and 72 had benign prostatic hypertrophy by histological findings.

tests and expressed as the prostate-specific antigen increment per year. Prostate-specific antigen velocity higher than $0.75 \mu \mathrm{g} / 1 \times$ year was suspicious for prostate cancer (18). In several cases, a negative velocity was recorded. If several prostate-specific antigen tests were performed during the previous year, a mean value of prostate-specific antigen velocity was calculated.

\section{Immunoassays}

Total and free prostate-specific antigen were measured using DPCs (Diagnostic Products Corporation, Los Angeles, CA) chemiluminescent enzyme immunoassays with the Immulite immunoanalyzer. Both DPC prostate-specific antigen tests are claimed to be in good agreement with Stanford International Standard by its manufacturer (33). Briefly, the Immulite prostate-specific antigen is a solid-phase, two-site chemiluminescent enzyme immunometric assay using a polyclonal anti-prostate-specific antigen as the capture antibody and a conjugated monoclonal as the detector antibody. The test's working range is $0.04-150 \mu \mathrm{g} / \mathrm{l}$.

The Immulite free prostate-specific antigen assay makes use of a monoclonal antibody specific for epitopes on prostate-specific antigen that are rendered inaccessible by complex formation between prostate-specific antigen and $\alpha_{1}$-antichymotrypsin as the capture antibody and a labelled polyclonal goat anti-prostate-specific antigen as the detector antibody. The free prostate-specific antigen assay has a working range of $0.05-100 \mu \mathrm{g} / \mathrm{l}$.

Both tests have good performance profiles as stated by the manufacturer and are monitored daily in our laboratory by internal and external quality control programmes. The inter- and intra-assay CV\% were $3.6 \%$ and $5.7 \%$ for total prostate-specific antigen and $3.5 \%$ and $6.0 \%$ for free prostate-specific antigen. The ratio of free/total prostate-specific antigen was calculated by dividing the free prostate-specific antigen duplicates mean value by the total prostate-specific antigen duplicates mean value and multiplied by 100 .

\section{Statistical analysis}

Wilcoxon (a non-parametric statistical test) was used to assess the statistical significance of differences in the values of total prostatespecific antigen and free/total prostate-specific antigen between benign prostatic hypertrophy and prostate cancer patients. (Receiveroperating characteristic (ROC) curves were generated by plotting sensitivity versus specificity to evaluate the free/total prostate-specific antigen assay performance.) Sensitivity, specificity, positive predictive value and negative predictive value were calculated for each parameter (total prostate-specific antigen, free/total prostatespecific antigen, prostate-specific antigen density, prostate-specific 
antigen velocity). Chi square statistical test was performed on each of the parameters against the benign/malignant diagnosis constant. Sensitivity, specificity and accuracy of combinations of different parameters were also calculated. A model of backwards logistic regression was applied in order to assess the relative weight of each of the parameters, while in each step the model eliminates the less significant parameter. The odds ratio represents the chances of having prostate cancer among the patients with positive tests, versus those with normal tests.

\section{Results}

We measured total and free prostate-specific antigen and calculated the proportion of free/total prostate-specific antigen in 3470 men aged $45-80$. In our institute only 147 men underwent prostate digital rectal examination, trans-rectal ultrasound guided, or 6 sextants systematic sampling of core biopsies of the prostate. Seventy-five of them indeed had biopsy-proven prostate cancer. Based on a previous pilot study (data unpublished), which calculated the sensitivity and specificity of free/total prostate-specific antigen at different cut-off levels, we plotted the ROC curve (fig. 2). The optimal cut-off point was found to be $11 \%$ with a sensitivity of $81 \%$ and a specificity of $43 \%$. A scattergram of the results is shown in figure 3.

Total prostate-specific antigen, free/total prostate-specific antigen, prostate-specific antigen velocity and prostate-specific antigen density are summarized for these patients in table 1 . The frequency of abnormal results is presented in tables $2 a$ and $2 b$.

Table 3 shows the sensitivities and specificities of each of the prostate-specific antigen parameters at the previously assigned cut-offs. Table 3 also adds the positive and negative predictive values, as well as the $P$ value of each of the prostate-specific antigen parameters.

Table 4a shows the estimated sensitivity, specificity and accuracy of combinations of the different prostate-specific antigen parameters, accomplished by using a back-

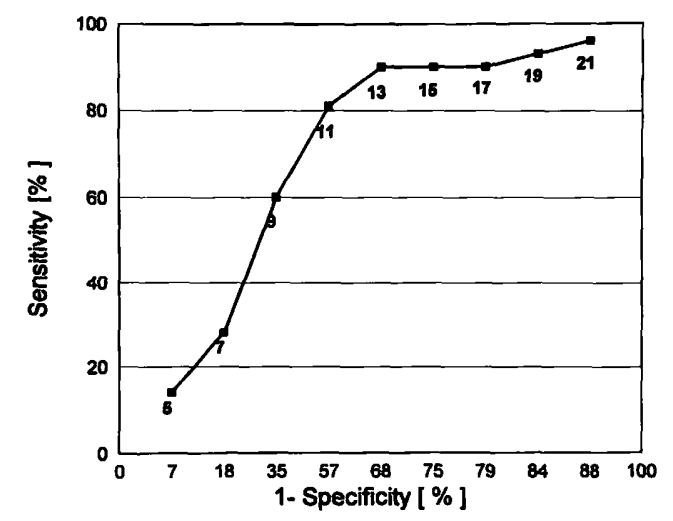

Fig. 2 Free/total prostate-specific antigen. Receiver operating characteristics analysis represents the sensitivity (\%) and 1-specificity (\%) of the test at different cut-off points. Points represent the different cut-offs $(5 \mu \mathrm{g} / 1$ to $21 \mu \mathrm{g} / \mathrm{l})$ at which sensitivities and specificities were calculated.
Tab. 1 Prostate-specific antigen parameters (age dependent total prostate-specific antigen, free/total prostate-specific antigen, prostate-specific antigen velocity and prostate-specific antigen density ) are represented by their mean, median and standard deviation, among the 72 benign prostatic hypertrophy and 75 prostate cancer patients.

(* = significant)

\begin{tabular}{llll}
\hline & $\begin{array}{l}\text { Benign } \\
\text { prostatic } \\
\text { hypertrophy }\end{array}$ & Prostate & P value \\
& & \\
$\mathrm{n}=72$ & $\mathrm{n}=75$ \\
& $\begin{array}{l}\text { Age (a) } \\
68.4 \pm 6.74\end{array}$ & Age (a) \\
& $70.8 \pm 9.07$ \\
\hline
\end{tabular}

Total prostate-specific

antigen $(\mu \mathrm{g} / \mathrm{l})$

$\begin{array}{lrc}\text { Mean } & 11.55 & 28.78 \\ \text { Median } & 9.20 & 9.80 \\ \text { SD } & 9.55 & 91.3\end{array}$

Free/total prostate-specific antigen (\%)

$\begin{array}{lrr}\text { Mean } & 12.34 & 9.56 \\ \text { Median } & 10.40 & 8.70 \\ \text { SD } & 7.19 & 5.43\end{array}$

$0.001^{*}$

Prostate-specific antigen

velocity $(\mu \mathrm{g} / 1 \times$ year $)$

$\begin{array}{lcr}\text { Mean } & 11.68 & 21.78 \\ \text { Median } & 3.7 & 9.90 \\ \text { SD } & 20.57 & 50.74\end{array}$

0.055

Prostate-specific antigen

density $(\mu \mathrm{g} / \mathrm{l} \times \mathrm{g})$

\begin{tabular}{llll} 
Mean & 0.34 & 1.27 & \\
Median & 0.26 & 0.36 & $0.001^{*}$ \\
SD & 0.30 & 3.95 & \\
\hline
\end{tabular}

ward logistic regression model. This model predicts the relative importance of each parameter. The model begins the evaluation with all parameters and eliminates in each step the quantity with the least significant contribution to the chi square, leaving eventually only the significant parameter. The results of the logistic regression are presented in table $4 \mathrm{~b}$. These results show the different prostate-specific antigen quantities by the order of their significance. Prostate-specific antigen density and total prostate-specific antigen were not significant and therefore have been withdrawn.

Figure 4 enables some observations regarding the capabilities of different parameter combinations. It is worth noting how misleading it could be to rely solely on positive total prostate-specific antigen and prostate-specific antigen density in predicting prostate cancer. It also shows the diagnostic improvement achieved by adding more parameter results. The figure shows that using all four parameters (adding free/total prostate-specific antigen to the total+velocity+density) increases the amount of prostate cancer patients by almost fivefold, while increasing a "wrongly" detected benign prostatic hypertrophy patient by 2.3 -fold. 
Tab. 2a The fraction of the abnormal results among all the 147 patients and its distribution in the benign prostatic hypertrophy and prostate cancer populations, in each of the prostate-specific antigen parameters.

\begin{tabular}{lllll}
\hline & $\begin{array}{l}\text { Total } \\
\text { prostate-specific } \\
\text { antigen }\end{array}$ & $\begin{array}{l}\text { Free/total } \\
\text { prostate-specific } \\
\text { antigen }\end{array}$ & $\begin{array}{l}\text { Prostate-specific } \\
\text { antigen velocity }\end{array}$ & $\begin{array}{l}\text { Prostate-specific } \\
\text { antigen density }\end{array}$ \\
\hline $\begin{array}{l}\text { Total fraction of abnormal results (\%) } \\
\begin{array}{l}\text { Abnormal results among benign prostatic } \\
\text { hypertrophy patients only (\%) }\end{array}\end{array}$ & 83.7 & 69.4 & 59.7 & 88.9 \\
$\begin{array}{l}\text { Abnormal results among prostate cancer } \\
\text { patients only (\%) }\end{array}$ & 86.1 & 56.9 & 39.0 & 46.7 \\
\begin{tabular}{l} 
P value (significant) \\
\hline
\end{tabular} & & 81.3 & 61.0 & 53.3 \\
\hline
\end{tabular}

Tab. 2b Focus on the interaction of total and free/total prostate-specific antigen results. The parenthesis represents the percentage of cancerous cases detected in these groups.

\begin{tabular}{lll}
\hline \multicolumn{1}{c}{ Free/total prostate-specific antigen } & $\begin{array}{l}\text { Abnormal } \\
(\%)\end{array}$ & $\begin{array}{l}\text { Normal } \\
(\%)\end{array}$ \\
\hline Total prostate-specific antigen & $69(59)$ & $24(24)$ \\
Abnormal & $9(67)$ & \\
\hline
\end{tabular}

Tab. 3 Sensitivity, specificity, positive predictive value, negative predictive value and $P$ value (Chi square test) for each of the 4 prostate-specific antigen parameters.

$*$ = non significant, $* *=$ significant.

\begin{tabular}{|c|c|c|c|c|c|}
\hline & (\%) & Specificity & $\begin{array}{l}\text { Positive } \\
\text { predictive } \\
\text { value } \\
(\%)\end{array}$ & $\begin{array}{l}\text { Negative } \\
\text { predictive } \\
\text { value } \\
(\%)\end{array}$ & $P$ value \\
\hline Total prostate-specific antigen & 81.3 & 13.9 & 49.6 & 41.7 & $0.433 *$ \\
\hline Free/total prostate-specific antigen & 81.3 & 43.1 & 59.8 & 68.9 & $0.001 * *$ \\
\hline Prostate-specific antigen velocity & 71.2 & 52.4 & 61.0 & 63.5 & $0.006 * *$ \\
\hline Prostate-specific antigen density & 94.1 & 16.4 & 53.3 & 73.3 & $0.051^{*}$ \\
\hline
\end{tabular}

Tab. 4a Predicted sensitivity, specificity, accuracy and significance ( $P$ value) of various prostate-specific antigen parameter combinations achieved by using a backward logistic regression model.

\begin{tabular}{|c|c|c|c|c|c|c|c|}
\hline \multicolumn{4}{|c|}{ Prostrate-specific antigen tests combined } & \multirow{2}{*}{$\begin{array}{l}\text { Sensitivity } \\
\text { (\%) }\end{array}$} & \multirow{2}{*}{$\begin{array}{l}\text { Specificity } \\
(\%)\end{array}$} & \multirow{2}{*}{$\begin{array}{l}\text { Accuracy } \\
\text { (\%) }\end{array}$} & \multirow[t]{2}{*}{$P$ value } \\
\hline Total & Free/total & Velocity & Density & & & & \\
\hline+ & + & + & + & 62.3 & 69.0 & 65.55 & 0.001 \\
\hline+ & + & + & - & 63.9 & 67.0 & 66.39 & 0.001 \\
\hline+ & - & + & + & 70.49 & 62.07 & 66.39 & 0.004 \\
\hline+ & + & - & + & 79.41 & 46.27 & 62.96 & 0.007 \\
\hline+ & + & - & - & 79.41 & 44.78 & 62.22 & 0.005 \\
\hline- & + & + & - & 54.1 & 75.86 & 64.71 & 0.0003 \\
\hline- & - & + & + & 62.21 & 63.79 & 65.55 & 0.002 \\
\hline
\end{tabular}

Tab. 4b First and final steps of the logistic regression represented by $P$ value and odds ratio of the examined parameters.

\begin{tabular}{llllll}
\hline & \multicolumn{2}{l}{ Step I - all parameters } & & & \multicolumn{2}{c}{ Final step } \\
\cline { 5 - 6 } \cline { 5 - 6 } & $\mathrm{P}$ & Odds ratio & & P & \multirow{2}{*}{ Odds ratio } \\
\hline Prostate-specific antigen velocity & 0.002 & 3.510 & 0.007 & 2.782 \\
Free/total prostate-specific antigen & 0.030 & 2.765 & & 0.016 & 2.678 \\
Total prostate-specific antigen & 0.174 & 0.457 & -- & -- \\
Prostate-specific antigen density & 0.441 & 1.760 & & \\
\hline
\end{tabular}




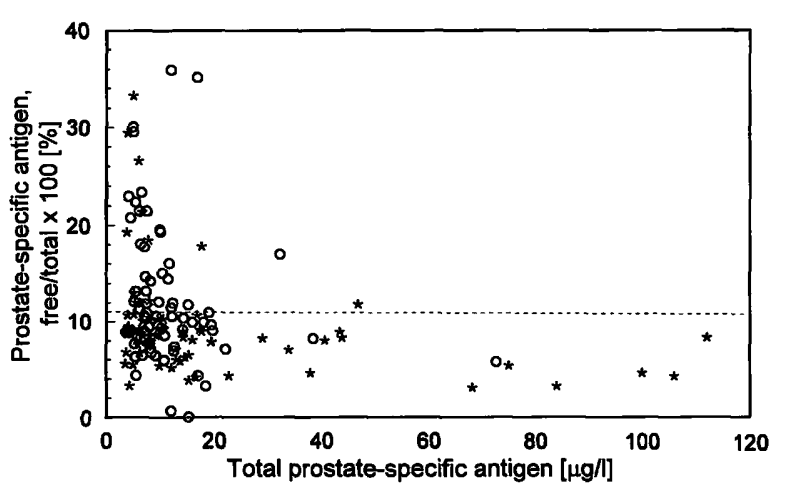

Fig. 3 A scattergram of all patients included in the study, free/total prostate-specific antigen is depicted as a function of the total prostate-specific antigen. $O=$ benign prostatic hypertrophy, $*=$ prostate cancer.

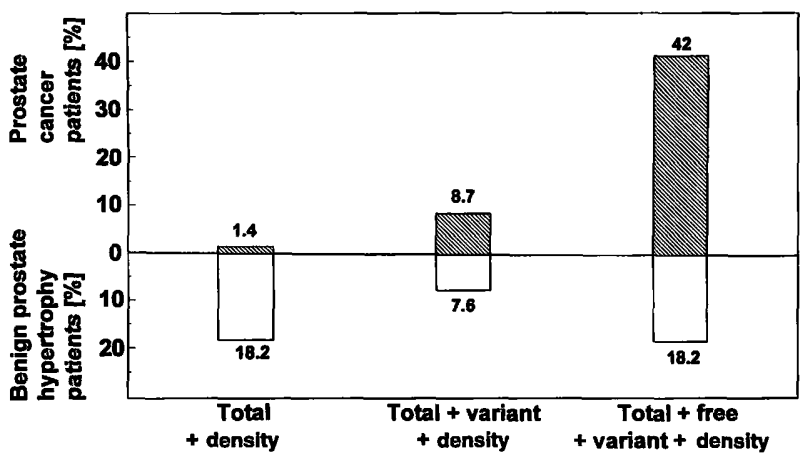

Fig. 4 Fraction of patients having positive prostate-specific antigen parameter combinations. The figure focuses on only three examples of combinations for reasons of simplifying the interpretation of the results.

\section{Discussion}

In the last three years there has been a significant increase in the general use of prostate-specific antigen by urologists and general practitioners. Today an abnormal prostate-specific antigen is probably the first step in the primary detection of prostate cancer in most of the prostate cancer patients. The considerably high rate of false-positive total prostate-specific antigen values led to the addition of prostate-specific antigen density and prostate-specific antigen velocity to the protocols of early prostate cancer detection in more recent works (15-19). In view of data indicating that free/total prostate-specific antigen enhances the specificity of the prostate-specific antigen test, it was added in the last four years to the battery of prostate-specific antigen parameters. Most of the studies concerning free/total prostatespecific antigen were performed retrospectively on stored sera of patients already diagnosed. The fact that free prostate-specific antigen was measured later than total prostate-specific antigen and/or later than the time of taking the fresh blood sample is even more significant in view of the data regarding the relative instability of the prostate-specific antigen- $\alpha_{1}$-antichymotrypsin complex $(38,39)$. In our study, total prostate-specific antigen and free prostate-specific antigen were performed simul- taneously on fresh blood samples, using one instrument, and kits of the same manufacturer.

Our goal in this study was to improve the accuracy of prostate-specific antigen for early detection of prostate cancer in patients with symptomatic prostatic disease. If prostate-specific antigen, with its various parameters, regardless and prior to using the other means for prostate cancer detection (digital rectal examination, trans-rectal ultrasound, biopsy, etc.), could render good diagnostic results it would mean saving and avoiding expensive and invasive and often unnecessary tests.

All the participants in our study had abnormal prostatespecific antigen values, either abnormal age-adjusted total prostate-specific antigen or abnormal free/total prostate-specific antigen: these were the inclusion criteria. Because of ethical reasons, patients with normal results were not offered a further investigative diagnostic procedure. The cut-off limit for free/total prostate-specific antigen was chosen to be $11 \%$ (free/total prostate-specific antigen $<11 \%$ indicative of cancer). This is based on our ROC curve (fig. 2) and is in agreement with Blankenstein's study (40) that uses the same kits and reached the same cut-off value. Our $11 \%$ cut-off value is lower than Oesterling's (29) or Christensson's 18\% (41), it therefore may enhance the specificity while lowering the sensitivity. The lower cut-off value is possibly due to a different calibration of the different kits as both in Blankenstein's and in our studies, different kits from those of Oesterling's and Christensson's were used. Recent data from another manufacturer (Boehringer-Mannheim) using their kits also indicate that the cut-off level should be $14 \%$ and not $18 \%$ (data on file - Boehringer-Mannheim).

Our inclusion criteria are clearly reflected by the frequency of abnormal marker results among the 147 examinees (tab. 2). It is clear that total prostate-specific antigen and prostate-specific antigen density are abnormal to the same extent and different than both free/total prostate-specific antigen and prostate-specific antigen velocity. These data correlate with various studies that demonstrated resemblance in the performance of the total prostate-specific antigen and prostate-specific antigen density. The high percentage of abnormal tests of total prostate-specific antigen and prostate-specific antigen density among all the 147 patients reflects a relatively high percentage of pathological results in the benign prostatic hypertrophy group in addition to the prostate cancer group. These data explain the low diagnostic accuracy of total prostate-specific antigen and prostatespecific antigen density as shown in our results. Table $2 b$ shows the distribution of total prostate-specific antigen positives and free/total prostate-specific antigen positives and how many of these had prostate cancer (and ignores the other quantities which were examined, namely the prostate-specific antigen velocity and the 
prostate-specific antigen density). It is important to note the diagnostic benefit of the free/total prostate-specific antigen over the total prostate-specific antigen, especially when considering the fact that these two tests would be, in most cases, the first step in the diagnostic work-up of a patient with prostate-related symptoms.

When each of the parameters (total prostate-specific antigen, free/total prostate-specific antigen, prostate-specific antigen density, prostate-specific antigen velocity) was examined separately, only free/total prostate-specific antigen and prostate-specific antigen velocity were statistically significant in detection of prostate cancer vs. benign prostatic hypertrophy, $\mathrm{P}$ value $<0.05$ (tab. 3). The inability of total prostate-specific antigen and prostate-specific antigen density to prove significance in detecting prostate cancer in contrast to benign prostatic hypertrophy does not undermine its value in detecting prostatic disease among normal patients, as shown in many studies. The latter two parameters failed, however, to prove significance in the given situation of an abnormal prostate-specific antigen test.

When logistic regression was performed (e. g. all four parameters are taken and then omitted one after the other if they fail to show significance, leaving only the significant parameters), a combination of free/total prostate-specific antigen and prostate-specific antigen velocity was reached. This combination has in fact a better $P$ value than any other single parameter or combination of parameters (tab. 4a). Another indication of the power of the free/total prostate-specific antigen and the prostate-specific antigen velocity combination can be found in the odds ratio calculation (tab. 4b), which shows a clear advantage of free/total prostate-specific antigen and prostate-specific antigen velocity over total prostate-specific antigen and prostate-specific antigen density.

As a single parameter, free/total prostate-specific antigen had the best $\mathrm{P}$ value $(0.0013)$. Free/total prostate-specific antigen had the best sensitivity $(81 \%)$, better than any single or combination of parameters.

Various other combinations, if only including either free/total prostate-specific antigen or prostate-specific antigen velocity, became also significant (tab. 4a). There is, in fact, no significant statistical difference between the $P$ values or the accuracies of the various combinations in table $4 \mathrm{a}$. The more substantial differences between these various combinations are different sensitivity, specificity and odds ratios.

The additional value of adding free/total prostate-specific antigen to the battery of total prostate-specific antigen, prostate-specific antigen density and prostate-specific antigen velocity is shown in figure 4 . When adding free/total prostate-specific antigen to the other three parameters the percentage of the prostate cancer patients increases (from $8.7 \%$ to $42 \%$ ), yet the "price" is doubling the amount of benign prostatic hypertrophy ("false positive" cases from $7.6 \%$ to $18.2 \%$ ). We claim that when dealing with a population at risk, the meaning of higher rates of early detection of prostate cancer is of greater importance than the cost and risk of the digital rectal examination, trans-rectal ultrasound and biopsy that would eventually be performed on benign prostatic hypertrophy patients.

We used the results of the core biopsies under transrectal ultrasound as our gold standard for detecting and establishing the diagnosis of prostate cancer vs. benign prostatic hypertrophy. It is reasonable to assume that there is a considerable amount of false negatives in these biopsies, and that a repeated biopsy taken after some time or a trans-urethral resection of prostate, prostatectomy or radical prostatectomy, would reveal more focuses of prostate cancer (with the latter being truly the gold standard for histologic diagnosis). The question whether such a bias is actually only for the best due to the latent nature and slow progression of the prostate cancer is still valid.

We propose an algorithm based on our findings and on clinical and practical considerations (fig. 5) for the use of the various prostate-specific antigen parameters in the early detection of prostate cancer. There is a need for ample further prospective studies incorporating this algorithm before its clinical benefit will be proven.

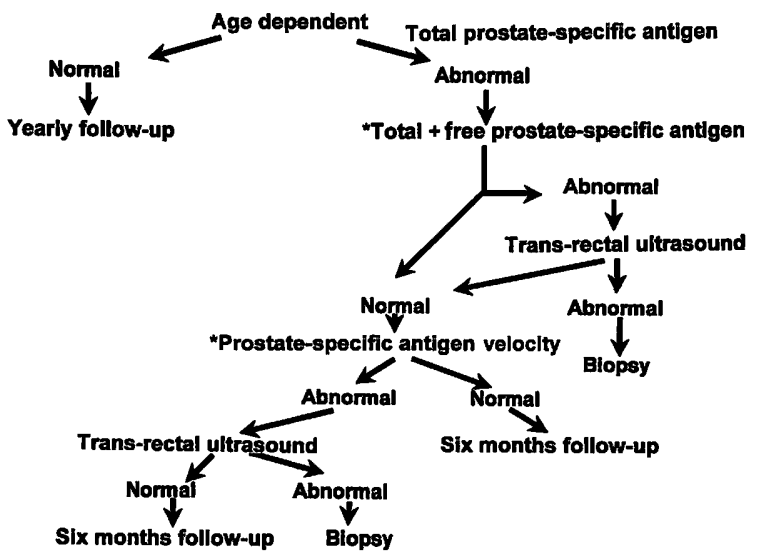

Fig. 5 Proposed algorithm for the use of prostate specific antigen parameters in early detection of prostate cancer. Each patient should undergo a yearly age-dependent total prostate-specific antigen examination. If abnormal, then total $(*-$ second time) and free prostate-specific antigen should be performed. If one of the two parameters is abnormal then trans-rectal ultrasound is required and is followed by biopsy if abnormal. If both total and free/total prostate-specific antigen are normal another (third) total prostatespecific antigen test is done to achieve the prostate-specific antigen velocity. If normal, a 6-monthly repeated follow up is offered. If the prostate-specific antigen velocity is abnormal trans-rectal ultrasound is required and is again followed by biopsy if abnormal, or only by a 6 -monthly follow-up if normal. 


\section{References}

1. Oesterling JE, Jacobsen SJ, Chute, CG, Guess HA, Girman CJ, Pauser LA, et al. Serum PSA in a community-based population of healthy men: establishment of age-specific reference ranges. J Am Med Ass 1993; 270:860-4.

2. Johansson JE, Adami H., Andersson SO, Bergstrom R, Holinberg L, Krusemo UB. High 10-year survival rate in patients with early, untreated prostate cancer. J Am Med Ass 1992; 267:2191-6.

3. Ohori M, Scordino PT. Early detection of prostate cancer: the nature of cancers detected with current diagnostic tests. Seminars in Oncol 1994; 21:522-6.

4. Schroder FH. Screening, early detection and treatment of prostate cancer, a European view. Urology 1995; 46 Suppl 3A:62-9.

5. Denis LJ. Prostate cancer screening and prevention: "realities and hope". Urology 1995; 46 Suppl 3A:56-61.

6. Quinlan DM, Partin AW, Walsh PC. Can aggressive prostate cancer be identified and can their natural history be altered by treatment? Urology 1995; 46:77-82.

7. Barak M, Mecz Y, Lurie A, Gruener N. Evaluation of PSA as a marker for adenocarcinoma of the prostate. J Lab Clin Med $1989 ; 13: 598-603$.

8. Catalona WT, Smith DS, Ratcliff TL, Dodds KM, Coplen DE, Yuan JJ, et al. Measurement of PSA in serum as a screening test for prostate cancer. N Eng J Med 1991; 324:1156-61.

9. Chadwick DJ, Kemple T, Astley JP. Pilot study of screening for prostate cancer in general practice. Lancet 1991; 338:613-6.

10. Brawer MK, Chetner MP, Beatie J, Buchner DM, Vessella RL, Lange $\mathrm{PH}$. Screening for prostate cancer with PSA. J Urol $1992 ; 147: 841-5$.

11. Labrie F, Dupont A, Subaru R. Serum PSA as pre-screening test for prostate cancer. J Urol 1992; 147:846-51.

12. Hudson MA, Bahnson RR, Catalona WJ. Clinical use of PSA in patients with prostate cancer. Urology 1989; 142:1011.

13. Lange PH, Ercole CJ, Lightner DJ, Fraley EE, Vessella R. The value of serum PSA determinations before and after radical prostatectomy. Urology 1989; 141:873.

14. Colberg J, Smith D, Catalona W. Prevalence and pathological extent of prostate cancer in men with PSA levels of 2.9 to 4.0 ng/ml. J Urol 1993; 149:507-9.

15. Benson MC, Whang IS, Pantuck A, Ring K, Kaplan SA, Olsson CA, Cooner WH. PSA density, a means of distinguishing benign prostatic disease and prostate cancer. J Urol 1992; 147:815-9.

16. Benson MC, Whang CS, Olsson CA, McMahon DJ, Cooner WH. The use of PSA density enhances the predictive value of intermediate levels of serum PSA. J Urol 1992; 147:815.

17. Seaman E, Whang IS, Olsson CA, Catz A, Cooner WH, Benson MC. PSA density (PSAD). Role in patient evaluation and management. Urol Clin N Am 1993; 20:653.

18. Carter HB, Pearson JD, Metter EJ, Brant LJ, Chan DW, Andres $\mathrm{R}$, et al. Longitudinal evaluation of PSA levels in men with and without prostate disease. J Am Med Ass 1992; 267:215.

19. Oesterling JE, Chute CG, Jacobsen SJ, Guess HA, Pauser LA, Johnson CL, et al. Longitudinal changes in serum PSA (PSA velocity) in a community-based cohort of men [abstract 799]. J Urol 1993; 149:412A.

20. Collins GN, Lee RJ, McKelvie GB, Rogers AC, Hehir M. Relationship between PSA, prostate volume and age in the benign prostate. Br J Urol 1993; 71:445.

21. Dolkin BL, Ahmann FR, Kopp JB. PSA levels in men older than 50 years without clinical evidence of prostate cancer. $\mathrm{J}$ Urol 1993; 150:1837.

22. Feneley MR, McLean A, Webb JAW, Kirby RS. Age corrected PSA in symptomatic benign prostatic disease [abstract 337]. $\mathrm{J}$ Urol 1994; 151:312A.

23. Christensson A, Laurell CB, Lilja H. Enzymatic activity of PSA and its reactions with extracellular serine protease inhibitors. Eur J Biochem 1990; 194:755.

24. Lilja H, Christensson A, Dahlen U, Matikainen MT, Nilsson $O$, Pettersson R, Largnen T. PSA in serum occurs predomi- nantly in complex with alpha-1-antichymotrypsin. Clin Chem $1991 ; 37: 1618-25$.

25. Zhou AM, Tewari PC, Bluestein BI, Caldwell GW, Larsen FL. Multiple forms of PSA in serum: differences in immunorecognition by monoclonal and polyclonal assays. Clin Chem 1993; 39:2483-91.

26. Petterson K, Piironen T, Seppela M, Linkkonen L, Christensson A, Matikainen MT, et al. Free and complexed PSA: in vitro stability, epitope map, and development of immunofluorimetric assays for specific and sensitive detection of free PSA and PSAal-antichymotrypsin complex. Clin Chem 1995; 41:1480-8.

27. Stenmman UH, Leionen J, Alfthan $H$, Rannikko S, Tuhkanen A, Alfthan U. A complex between PSA and alpha-1-antichymotrypsin is the major form of PSA in serum of patients with prostate cancer: assay of the complex improves the clinical sensitivity for cancer. Cancer Res 1991; 51:222-6.

28. Stenmman UH. Hakama M, Knekt P, Aromaa A, Teppo L, Leinonen J. Serum concentrations of PSA and its complex with $\alpha$ 1-antichymotrypsin before diagnosis of prostate cancer. Lancet 1994; 344:1594-8.

29. Oesterling JE, Jacobsen SJ, Klee GG, Pettersson K, Piironen $T$, Abrahamsson PA, et al. Free, complexed and total serum PSA: the establishment of appropriate reference ranges for their concentrations and ratios. J Urol 1995; 154:1090-5.

30. Luderer AA, Chen YT, Soriano TF, Kramp WJ, Carlson G, Lung $C$, et al. Measurement of the proportion of free to total PSA improves diagnostic performance of PSA in the diagnostic gray zone of total PSA. Urology 1995; 46:187-94.

31. Catalona WJ, Smith DS, Wolfert RL, Wang TJ, Rittenhouse HG, Ratcliff TL, et al. Evaluation of percentage of free serum PSA to improve specificity of prostate cancer screening. J Am Med Ass 1995; 274:1214-20.

32. Terris MK, Stamey TA. Determination of prostate volume by transrectal ultrasound. J Urol 1991; 145:984-7.

33. Murphy GP. The second Stanford conference on international standardization of PSA assays. Cancer 1994; 75:122-8.

34. Catalona WJ, Richie JP, Ahmann FR, Hudson MA, Scardino PT, Flanigan RC, et al. Comparison of digital-rectal examination and serum prostate specific antigen in the early detection of prostate cancer: results of multicenter clinical trial of 6,630 men. J Urol 1994; 151:1283-90.

35. Catalona WJ, Smith DS, Ratcliff TL, Dodds KM, Coplen BE, Yuan JJ, et al. Measurement of prostate specific antigen in serum as a screening test for prostate cancer. $N$ Engl $J$ Med $1991 ; 324: 1156-61$.

36. Brawer MK, Chatner MP, Beatie J, Buchner DM, Vessella RL, Lang $\mathrm{PH}$, et al. Screening for prostatic carcinoma with prostate specific antigen. J Urol 1992; 147:841-5.

37. Mettlin C, Littrup PJ, Kane RA, Murphy GP, Lee F, Chesley $A$, et al. Relative sensitivity and specificity of serum PSA level compared with age-referenced PSA, PSA density and PSA change. Cancer 1994; 74:1615-20.

38. Leionen J, Zhang WM, Stennman UH. Complex formation between PSA isoenzymes and protease inhibitors. J Urol 1996; 155:1099-103.

39. Chen Z, Prestigiacomo A, Stamey TA. Purification and characterization of PSA complexed to $\alpha_{1}$-antichymotrypsin: potential reference material for international standardization of PSA immunoassays. Clin Chem 1995; 41:1273-82.

40. Blankenstein MA, van Aubel OGJM, van Zon J, Veldkamp A. Preliminary and clinical evaluation of the DPC Immulite assay for free PSA. Tumor Biol 1996; 1 Suppl 17:21.

41. Christensson A, Bjork $T$, Nielson $O$, Dahlen U, Matikainen MT, Cockett AT, et al. Serum PSA compared to $\alpha_{1}$-antichymotrypsin as an indicator of prostate cancer. J Urol 1993; 150:100-5.

Received October 10, 1996/March 4, 1997

Corresponding author: Mira Barak, Ph.D., General Director, Biochemistry Tumor-Markers, Haifa and Western Galilee Central Laboratory, 47 Hataasia, P. O. Box 9107, Nesher, Israel 
